\title{
AVALIAÇÃO DE DUAS METODOLOGIAS DE EXPANSÃO AO FORNO DO POLVILHO AZEDO' ${ }^{1}$
}

\author{
Kelly C. MAEDA², Marney P. CEREDA ${ }^{3, *}$
}

\begin{abstract}
RESUMO
Duas metodologias de expansão ao forno foram comparadas em 52 amostras de polvilho azedo de três Estados: Santa Catarina, Paraná e Minas Gerais. Os resultados obtidos permitiram visualizar uma proposta de classificação em nível nacional da expansão ao forno do polvilho azedo. No método instrumental foi usado o Farinógrafo Brabender para estabelecer uma massa de consistência padrão de 60UF e no método prático, a consistência semelhante da massa foi determinada de forma empírica. Com a metodologia instrumental obteve-se biscoitos com expansão superior à metodologia prática, mas maior coeficiente de variação, o que pode ser devido a maior sensibilidade do método. Ambas as metodologias poderão ter aplicação, dependendo da finalidade. A metodologia instrumental, pelo custo do equipamento, é mais adequada para laboratórios, desde que se utilize maior número de repetições, para reduzir o coeficiente de variação. Ambas as metodologias ensaiadas permitiram classificar as amostras com o mesmo perfil de desempenho. As amostras provenientes do Estado do Paraná apresentaram as maiores expansões ao forno quando comparados com os outros Estados. Pela metodologia prática a expansão média das amostras do Estado do Paraná foi de $14,1 \mathrm{~mL} / \mathrm{g}$. As amostras do Estado de Minas Gerais apresentaram as menores expansões ao forno, em média de $13,3 \mathrm{~mL} / \mathrm{g}$. Foi proposta neste artigo uma classificação para avaliar a qualidade do polvilho azedo, complementando a legislação brasileira que não estabelece este índice para o produto. Quanto à propriedade de expansão ao forno, as amostras avaliadas podem ser divididas em 3 tipos A, B e C. No tipo A (10\%) amostras de polvilhos azedo são classificadas como qualidade extra, no tipo B $(80 \%)$ os polvilhos são de média qualidade e o tipo C (10\%) de qualidade inferior. Os resultados obtidos pela metodologia prática permitiram estabelecer como tipo $\mathrm{A}$ o polvilho azedo que apresenta um índice de expansão ao forno superior a $16,0 \mathrm{~mL} / \mathrm{g}$, o tipo B o polvilho com índice de expansão entre $12,0 \mathrm{~mL} / \mathrm{g}$ a $16,0 \mathrm{~mL} / \mathrm{g}$ e o tipo $\mathrm{C}$ o polvilho com índice de expansão menor que $12,0 \mathrm{~mL} / \mathrm{g}$.
\end{abstract}

Palavras-chave: polvilho azedo; expansão; qualidade; classificação.

\section{SUMMARY}

EVALUATION OF TWO METHODOLOGIES OF EXPANSION IN OVEN OF SOUR CASSAVA STARCH. Two methodologies for measuring the expansion in oven were compared in 52 samples of fermented cassava starch of three States: Santa Catarina, Paraná and Minas Gerais. The results allowed a classification for the product based in the expansion in oven as quality evaluation. The instrumental method of Brabender Farinograph was used to establish standard dough with 60FU and in the practice method

\footnotetext{
${ }_{1}^{1}$ Recebido para publicação em 15/06/99. Aceito para publicação em 19/07/01.

${ }^{2}$ Mestre em Ciência e Tecnologia de Alimentos, ESALQ - USP, Cx. Postal 9, CEP 13418-900, Piracicaba-SP, Brasil.

${ }^{3}$ Pesquisador do Centro de Raízes e Amidos Tropicais, CERATUNESP, Cx. Postal 237, CEP 18603-970, Botucatu - SP, Brasil. E-mail: cereda@terra.com.br e seccerat@fca.unesp.br *A quem a correspondência deve ser enviada.
}

the appropriate consistency of the dough was adjusted by an empirical way. Instrumental methodology obtained bigger expansions than with the practical methodology, but larger variation coefficient was observed due to larger sensibility of this method. Both methodologies can have applications depending on the purpose. The instrumental methodology is of a high cost mainly because of the Brabender Farinograph price but also because of the larger number of repetitions necessary to reduce the variation coefficient. Both methodologies allowed classifying the samples with the same profile. Samples coming from the State of Paraná presented the largest expansions in the oven when compared with samples of other States. Using practical methodology the medium expansion of the samples of the State of Paraná was of $14,1 \mathrm{~mL} / \mathrm{g}$. Samples of the State of Minas Gerais presented the smallest expansions in the oven, on the average of $13,3 \mathrm{~mL} / \mathrm{g}$. This paper proposes a classification as a form of evaluating the quality of the fermented cassava starch, complementing the Brazilian Legislation that doesn't establish this index for the product. With relationship to the expansion property to the oven, the samples can be divided in 3 types. In type $A(10 \%)$ of fermented cassava starch samples are classified as extra quality, type B $(80 \%)$ are of medium quality and type $C$ $(10 \%)$ of inferior quality. The results obtained by the practical methodology allowed establishing type A presenting an expansion index upper $16,0 \mathrm{~mL} / \mathrm{g}$, B type expansion index among $12,0 \mathrm{~mL} / \mathrm{g}$ to $16,0 \mathrm{~mL} / \mathrm{g}$ and $C$ type with expansion bellow $12,0 \mathrm{~mL} / \mathrm{g}$.

Keywords: fermented cassava starch; expansion index in oven; quality; classification of the sour cassava starch.

\section{1 - INTRODUÇÃO}

A fécula de mandioca recebe também a denominação de polvilho. O polvilho azedo é um tipo de fécula de mandioca modificado por processo de fermentação e secagem solar, apresentando características bem diversas do polvilho doce. O polvilho azedo é um produto regional e embora de preparo artesanal, a sua produção já era grande em 1978, nos Estados de Minas Gerais, Santa Catarina, Paraná e São Paulo [14], onde é fabricado por um grande número de indústrias rurais de pequeno porte. VILPOUX [15] baseando-se em dados recolhidos no final de 1996, em entrevistas com 26 empresários e entidades de pesquisa e extensão nas diferentes regiões de produção observou que a produção brasileira de polvilho azedo cresceu bastante, principalmente devido ao aumento de consumo de pão-dequeijo. Tradicionalmente, o polvilho azedo era produzido nos Estados de Minas Gerais e Santa Catarina, na região vizinha ao Rio Grande do Sul, mas a produção do Estado do Paraná cresceu muito, ocupando uma fatia de mercado cada vez maior. Devido à necessidade de diversificação da produção das fecularias, o polvilho azedo oferece-se como uma alternativa de baixo custo à produção de amidos modificados. Frente à crise das empresas de farinha muitas indústrias aspiram a uma conversão da planta de processamento para produção de polvilho. Essa solução tem a vantagem de aprovei- 
tar parte da estrutura da farinheira e de não exigir investimentos muito elevados. Isso está atraindo cada vez mais empresários para a produção de polvilho azedo. Segundo CEREDA [7] a procura é grande pelo consumidor, principalmente para ser utilizado em produtos de confeitaria na forma de biscoitos, sequilhos, pão-dequeijo, bolos, etc. É insubstituível no preparo de biscoito salgado que se caracteriza por ser um produto muito leve e volumoso, resultado da expansão do polvilho azedo no forno. A confecção do biscoito de polvilho é, até o momento, o único teste de qualidade existente aceito por todos, usuários e produtores. Esse fato justifica a busca de um índice de qualidade e já existem estudos de duas metodologias que analisam a expansão ao forno do polvilho azedo comercial. A metodologia prática, segundo NUNES \& CEREDA [11], é uma análise de baixo custo acessível ao produtor e aos pequenos e médios fabricantes que não dispõem de equipamentos mais sofisticados. Necessita-se apenas de uma pessoa treinada para realizar a análise. Já a metodologia instrumental adaptada por CEREDA [5] da AMERICAN ASSOCIATION OF CEREAL CHEMISTS [1] e por NUNES \& CEREDA [11], utiliza o Farinógrafo Brabender e tem como base uma formulação padrão para biscoitos estabelecida por CEREDA [4]. É uma metodologia mais demorada que necessita treinamento especializado do analista e o custo do equipamento é muito elevado.

$\mathrm{Na}$ legislação não existe ainda uma classificação para polvilho azedo segundo os índices de expansão ao forno. Como a análise de expansão ao forno é importante para estabelecer a qualidade do polvilho azedo, neste trabalho foram comparadas 52 amostras de polvilho azedo dos três principais Estados produtores brasileiros: Paraná, Santa Catarina e Minas Gerais. O índice de expansão ao forno, em volume específico $(\mathrm{mL} / \mathrm{g})$, foi determinado utilizando-se duas metodologias, a prática e a instrumental.

Segundo CEREDA [7], o polvilho azedo é obtido pela fermentação de polvilho doce, podendo também ser produzido da fécula recuperada do líquido de prensagem da massa ralada, como sub-produto da fabricação de farinha de mandioca e de raspas. Após a fermentação, o polvilho azedo é submetido à secagem ao sol. Produtores que tentaram secagem artificial asseguram que não obtiveram produto seco com o mesmo poder de expansão, sugerindo que mais que o calor, é a radiação solar a responsável pela propriedade. Para confirmar esta hipótese CEREDA \& NUNES [8] utilizaram polvilho azedo fabricado na indústria Caribé, no município de Conceição dos Ouros, Estado de Minas Gerais, com 28 dias de fermentação. As amostras foram submetidas à secagem em "Flash Dryer" e natural. O melhor resultado de expansão ao forno foi obtido no polvilho azedo seco ao sol das 9:30 às 17:00 horas com um valor médio de $10,69 \mathrm{~mL} / \mathrm{g}$. Mestres, ZAKHIA \& DUFOUR [10], seguindo a mesma metodologia adotada por CEREDA \& NUNES [8], avaliaram a expansão ao forno de fécula de mandioca colombiana após submeter as amostras aos seguintes tratamentos: polvilho doce seco à sombra, seco em estufa e ao sol. A habili- dade de expansão ao forno ou expansibilidade do polvilho azedo seco ao sol foi duas vezes maior que nas outras condições de secagem. Os resultados obtidos foram de $6,3 \mathrm{~mL} / \mathrm{g}$ para o polvilho doce, $7,0 \mathrm{~mL} / \mathrm{g}$ para o polvilho azedo seco em estufa e $14,9 \mathrm{~mL} / \mathrm{g}$ para o polviIho azedo seco ao sol. A expansão do polvilho azedo seco ao sol foi de $16,7 \mathrm{~mL} / \mathrm{g}$ quando o $\mathrm{pH}$ chegou a 4 . De acordo com os autores, a fermentação e a secagem solar são necessárias para conferir a habilidade de expansão ao forno da fécula de mandioca.

A expansão ao forno do polvilho azedo é diferente da expansão dos grânulos de amido, que também incham ou entumescem em suspensão aquosa. Os trabalhos prévios de CEREDA [4] mostraram que nas mesmas condições as pastas de polvilho doce (não fermentado e exposto ao sol) não se expandem ao forno.

Segundo alguns autores a expansão do polvilho azedo pode depender de outros fatores. BRABET et al [3] citados por NUNES [12] isolaram bactérias lácticas, produtoras de exopolissacarídeos (EPS), que seriam responsáveis pela formação de uma estrutura viscoelástica, a qual permitiria a retenção de gás e expansão da massa de polvilho azedo durante o forneamento.

CEREDA [4], partindo da hipótese de que a qualidade do polvilho azedo está relacionada ao seu desempenho no forno, estabeleceu uma formulação básica para o biscoito de polvilho, com pequeno número de variáveis à semelhança do que existe para a panificação experimental. A formulação padrão consiste em polvilho azedo $100 \%$, gordura hidrogenada $25 \%$, sal $4 \%$ e água ao redor de $80 \%$ (variável de acordo com o polvilho azedo utilizado). A massa foi desenvolvida em uma batedeira e a consistência determinada em consistômetro Bostwick. Os biscoitos foram confeccionados com auxílio de um confeitador diretamente sobre assadeira, que foi levada ao forno a $200^{\circ} \mathrm{C}$ por 10 a 20 minutos. Com esta formulação e metodologia, CEREDA [6] avaliou a qualidade de duas amostras de polvilho azedo comercial denominadas de A (proveniente do Estado do Paraná) e B (proveniente do Estado de São Paulo). $O$ polvilho $A$ apresentou nos ensaios de forneamento, maior volume específico, de $16,68 \mathrm{~mL} / \mathrm{g}$ no primeiro e $10,70 \mathrm{~mL} / \mathrm{g}$ no segundo ensaio. Para o polvilho azedo $\mathrm{B}$ os valores foram de $4,71 \mathrm{~mL} / \mathrm{g}$ para o primeiro ensaio e $4,64 \mathrm{~mL} / \mathrm{g}$ para o segundo ensaio. NUNES \& CEREDA [11] estabeleceram índices para classificar a expansão em pequena $\leq 5,0 \mathrm{~mL} / \mathrm{g}$, média de 5,0 a $10 \mathrm{~mL} / \mathrm{g}$ e grande $\geq 10,0 \mathrm{~mL} / \mathrm{g}$.

PLATA OVIEDO \& CAMARGO [13] avaliaram amostras de polvilho azedo Cruzeiro do Sul e Caribé, através do teste de biscoito seguindo formulação padrão descrita por CEREDA [4], com redução do sal para $3 \%$. O menor volume específico foi obtido para o polvilho azedo Cruzeiro do Sul $(11 \mathrm{~mL} / \mathrm{g})$, em relação ao Caribé $(15 \mathrm{~mL} / \mathrm{g})$.

DEMIATE et al [9] analisaram amostras de polvilho azedo coletadas junto ao comércio no Estado do Paraná. A propriedade de expansão foi avaliada utilizando-se a metodologia e a formulação de biscoitos descrita por 
CEREDA [4]; o volume específico médio encontrado foi de $7,66 \mathrm{~mL} / \mathrm{g}$.

\section{2 - MATERIAL E MÉTODOS}

\section{1 - Matéria-prima}

Para a realização deste trabalho foram utilizadas 52 amostras de $1,0 \mathrm{~kg}$ de polvilho azedo comercial, obtidas diretamente de indústrias dos Estados de Santa Catarina, Paraná e Minas Gerais na safra de 1996.

\section{2 - Métodos}

A propriedade de expansão do polvilho azedo foi avaliada através do teste de expansão ao forno dos biscoitos seguindo duas metodologias de expansão, a metodologia instrumental e a metodologia prática.

\subsection{1 - Metodologia instrumental}

Foi utilizada a formulação padrão desenvolvida por CEREDA [4] com algumas modificações: polvilho azedo $(100 \%)$ e os demais ingredientes calculados como porcentagem sobre o peso do polvilho. A quantidade de gordura vegetal hidrogenada foi reduzida de $25 \%$ para $20 \%$, sal (4\%) e água ao redor de $80 \%$, variável em função da amostra.

A metodologia seguiu a descrição de CEREDA \& NUNES [8] modificada. Para confeccionar-se os biscoitos foi utilizado Farinógrafo Brabender. O polvilho azedo inicialmente foi colocado no copo do aparelho, mantido a $30^{\circ} \mathrm{C}$ e regulado para $62,5 \mathrm{rpm}$ e escala de 1000 Unidades Farinográficas (UF). O aparelho funcionou por 5 minutos para homogeneização da amostra. A seguir, adicionou-se $10 \mathrm{~mL}$ de água fria e o aparelho funcionou por mais 5 minutos. Sem desligar o mesmo, foi feito o escaldamento do material, utilizando-se $10 \mathrm{~mL}$ da mistura fervente $\left(97^{\circ} \mathrm{C}\right)$ de água destilada, sal e gordura vegetal. Homogeneizou-se a mistura por mais 10 minutos e depois se acrescentou água destilada fria, lentamente à massa até obtenção de consistência 60UF, consistindo esta em modificação da metodologia anterior que propunha 40UF. A massa retirada do Farinógrafo foi usada para confeccionar os biscoitos, padronizados em $8,0 \mathrm{~cm}$ de comprimento e $0,5 \mathrm{~cm}$ de diâmetro. Para isso, foi utilizado um confeitador com bico redondo. Os biscoitos foram assados em forno termoestatizado marca Layr, modelo SA 4259 a $200^{\circ} \mathrm{C}$ por 10 a 15 minutos. Os biscoitos confeccionados pelo método instrumental depois de frios foram pesados e os volumes de cada biscoito determinados pelo método de deslocamento de sementes [2]. Para estes biscoitos foram utilizadas sementes de painço e então, transbordamento e leitura volumétrica. Obteve-se o volume específico (expansão) expresso em $\mathrm{mL} / \mathrm{g}$ calculado pela relação entre o volume $(\mathrm{mL})$ e o peso $(\mathrm{g})$ de cada biscoito. A expansão das amostras foi comparada aos índices de expansão estabelecidos por NUNES \& CEREDA [11] para classificar o polvilho azedo em pequeno $(\leq 5,0 \mathrm{~mL} / \mathrm{g}$ ), médio (de $5,0$ a $10 \mathrm{~mL} / \mathrm{g})$ e grande $(\geq 10,0 \mathrm{~mL} / \mathrm{g})$.

\subsection{2 - Metodologia prática}

Foram pesados $50 \mathrm{~g}$ de polvilho azedo e sobre a amostra colocados aproximadamente $40 \mathrm{~mL}$ de água fervente [11]. A massa foi modelada, testando-se a consistência nas mãos até tornar-se homogênea e macia suficiente para ser moldada. Caso permanecesse dura e quebradiça, um pouco mais de água fervente era adicionado até obter-se a consistência ideal. Com a massa modelada foram confeccionados cinco biscoitos redondos de aproximadamente 10 gramas cada. Foram distribuídos em assadeira e levados ao forno elétrico termoestatizado marca Layr, modelo SA 4259 à temperatura de $200^{\circ} \mathrm{C}$, por 25 minutos. Os volumes dos biscoitos foram determinados pelo mesmo método descrito na metodologia instrumental e os volumes específicos $(\mathrm{mL} / \mathrm{g})$ comparados aos índices de expansão [11].

\section{3 - RESULTADOS E DISCUSSÃO}

\section{1 - Metodologias prática e instrumental de expan- são ao forno}

Todos os resultados de expansão ao forno (Tabela 1) obtidos pelas metodologias prática e instrumental, podem ser classificados através dos índices estabelecidos por NUNES \& CEREDA [11] como de grande expansão, com resultados superiores a $10,0 \mathrm{~mL} / \mathrm{g}$. Comparando-se os resultados dos índices de expansão ao forno, observa-se que pela metodologia instrumental foram obtidas as maiores médias, de $21,4 \mathrm{~mL} / \mathrm{g}$ para as amostras do Estado do Paraná, $18,6 \mathrm{~mL} / \mathrm{g}$ para Santa Catarina e $17,0 \mathrm{~mL} / \mathrm{g}$ para Minas Gerais. A média geral foi de $18,8 \mathrm{~mL} / \mathrm{g}$. Os resultados médios dos índices de expansão ao forno obtidos pela metodologia prática foram menores que os resultados obtidos pela metodologia instrumental. Através dos resultados de variância obtidos para o conjunto de todas as amostras observase, que a variância para a metodologia prática foi menor, que os resultados obtidos por metodologia instrumental. A maior variância observada na metodologia instrumental pode ser devida à maior sensibilidade do método, exigindo para maior confiabilidade um número maior de repetições. Entretanto o elevado custo do equipamento restringe o uso desta metodologia para laboratórios especializados, enquanto que a metodologia prática pode ser usada pelos produtores para acompanhar a qualidade do produto.

Como o polvilho azedo é obtido a partir de fermentação natural e secagem solar e ainda hoje produzido por grande número de pequenas indústrias rurais, torna-se difícil obter um produto de qualidade padronizada. Como a legislação brasileira não exige um índice específico para classificar a expansão ao forno do polvilho azedo, foi proposta neste trabalho uma classificação em 3 tipos. O tipo A abrange os polvilhos de qualidade extra, o tipo $B$ de média qualidade e o tipo $C$ de qualidade inferior. A proposta de classificação parte do princípio de atender a maior parte da produção brasileira de polvilho azedo. Portanto $80 \%$ do polvilho azedo 
nacional faria parte da classificação tipo $B$, sendo os outros $20 \%$ igualmente divididos entre os tipos A e C.

TABELA 1. Resultados dos índices de expansão ao forno obtidos através das metodologias prática e instrumental e análise estatística, expressos em $\mathrm{mL} / \mathrm{g}$ em amostras de polviIho azedo dos Estados de Santa Catarina, Minas Gerais e Paraná coletadas em 1996.

\begin{tabular}{|c|c|c|c|c|c|c|c|c|c|c|c|c|c|c|}
\hline \multicolumn{9}{|c|}{ SANTA CATARINA } & \multicolumn{3}{|c|}{ MINAS GERAIS } & \multicolumn{3}{|c|}{ PARANÁ } \\
\hline$P A$ & MP & MI & $P A$ & MP & MI & $P A$ & MP & MI & $P A$ & MP & MI & $P A$ & MP & MI \\
\hline 1 & 14,7 & 19,3 & 13 & 14,9 & 16,0 & 25 & 12,7 & 18,2 & 1 & 10,5 & 18,9 & 1 & 13,6 & 16,4 \\
\hline 2 & 13,6 & 17,9 & 14 & 12,4 & 14,9 & 26 & 15,7 & 25,9 & 2 & 11,6 & 14,5 & 2 & 14,8 & 24,6 \\
\hline 3 & 10,1 & 17,4 & 15 & 15,7 & 16,8 & 27 & 12,2 & 23,9 & 3 & 12,8 & 13,4 & 3 & 16,5 & 20,5 \\
\hline 4 & 15,0 & 18,3 & 16 & 15,5 & 17,8 & 28 & 13,5 & 16,6 & 4 & 14,8 & 16,4 & 4 & 12,2 & 24,6 \\
\hline 5 & 13,0 & 14,2 & 17 & 13,8 & 16,3 & 29 & 13,3 & 13,0 & 5 & 14,9 & 20,3 & 5 & 13,0 & 25,0 \\
\hline 6 & 12,4 & 18,5 & 18 & 12,9 & 19,0 & 30 & 13,9 & 26,0 & 6 & 12,9 & 13,4 & 6 & 13,6 & 22,6 \\
\hline 7 & 12,8 & 15,6 & 19 & 13,9 & 21,1 & 31 & 14,9 & 18,3 & 7 & 14,2 & 18,7 & 7 & 13,7 & 22,9 \\
\hline 8 & 13,9 & 17,9 & 20 & 12,9 & 18,5 & 32 & 14,5 & 25,6 & 8 & 14,8 & 20,2 & 8 & 15,0 & 14,8 \\
\hline 9 & 15,4 & 16,9 & 21 & 13,9 & 19,0 & 33 & 14,8 & 20,2 & & & & & & \\
\hline 10 & 13,6 & 16,9 & 22 & 15,7 & 25,0 & 34 & 15,8 & 21,2 & & & & & & \\
\hline 11 & 12,3 & 12,7 & 23 & 16,3 & 19,5 & 35 & 16,6 & 20,2 & & & & & & \\
\hline 12 & 14,0 & 14,0 & 24 & 15,2 & 17,8 & 36 & 15,9 & 19,4 & & & & & & \\
\hline \multicolumn{2}{|c|}{ Média } & & & & & & 14,1 & 18,6 & & 13,3 & 17,0 & & 14,1 & 21,4 \\
\hline \multicolumn{2}{|c|}{ Mediana } & & & & & & 13,9 & 18,2 & & 13,5 & 17,5 & & 13,7 & 22,8 \\
\hline \multicolumn{2}{|c|}{ Variância } & & & & & & 2,0 & 11,5 & & 2,7 & 8,5 & & 1,8 & 15,2 \\
\hline
\end{tabular}

$\mathrm{PA}=$ polvilho azedo $\mathrm{MP}=$ método prático $\mathrm{MI}=$ método instrumental

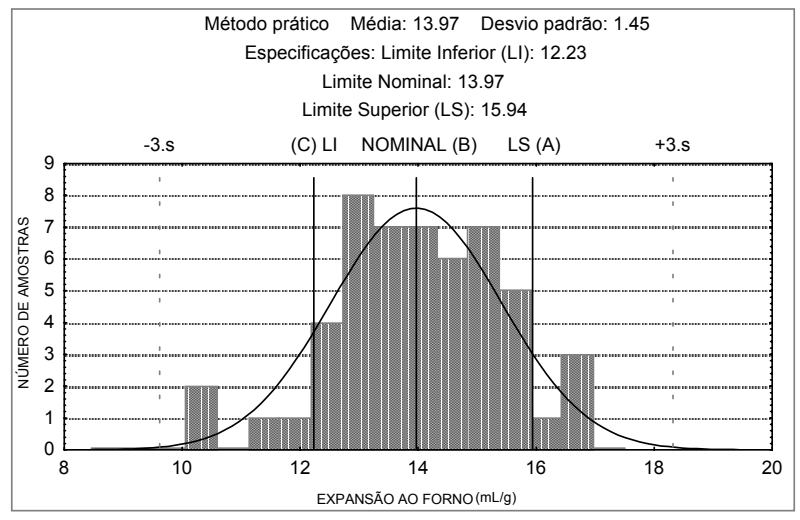

(a)

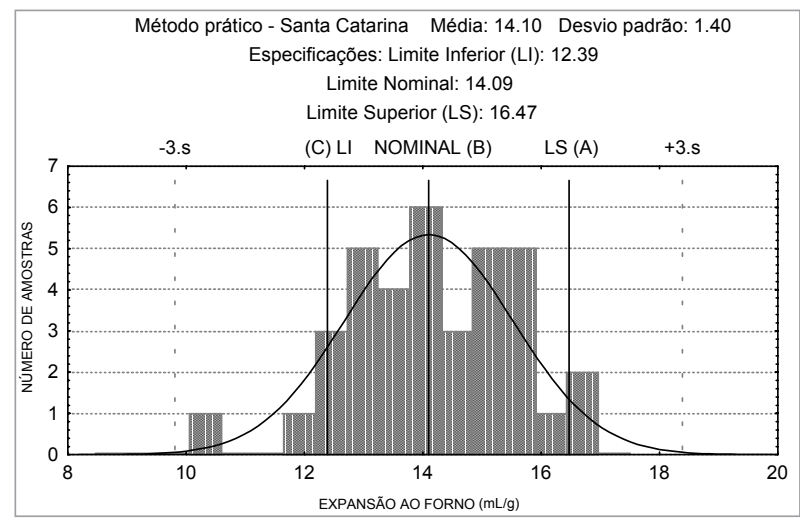

(b)

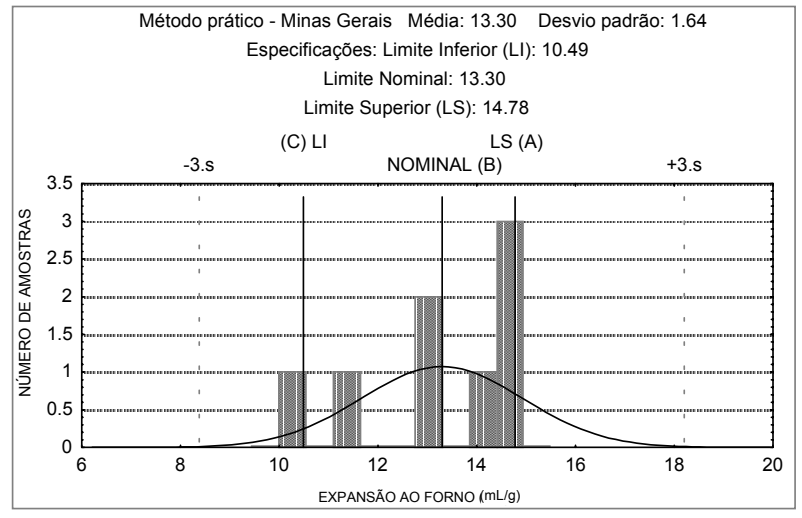

(c)

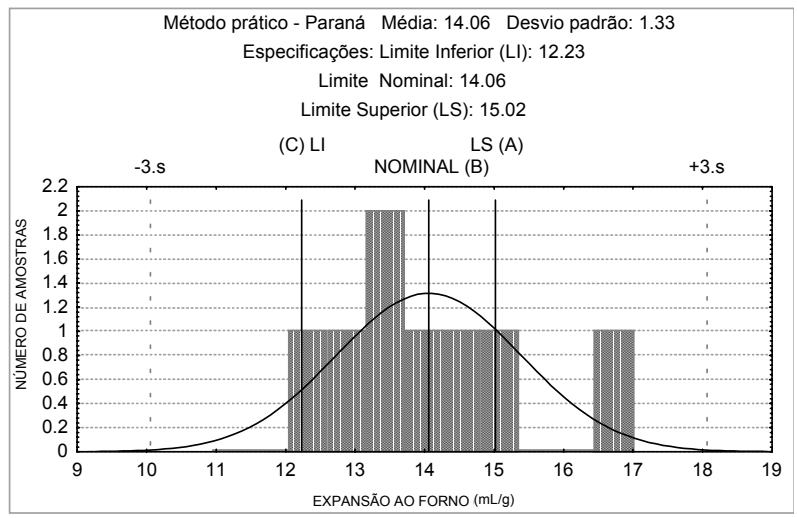

(d)

FIGURA 1. Resultados de expansão ao forno do polvilho azedo pelo método prático $(\mathrm{mL} / \mathrm{g})$ da classificação em tipos $\mathrm{A}, \mathrm{B}$ e C: geral das amostras (a), do Estado de Santa Catarina (b), Minas Gerais (c) e Paraná (d).

A proposta de classificação (Figura 1 (a)) foi baseada nos índices de expansão ao forno obtidos pela metodologia prática, mais fácil de ser introduzida nas empresas para controle da qualidade do produto. $\mathrm{O}$ tipo A apresentou amostras com índice de expansão ao forno superior a $16,0 \mathrm{~mL} / \mathrm{g}$, o tipo $B$, entre $12,2 \mathrm{~mL} / \mathrm{g} \mathrm{e}$ $16,0 \mathrm{~mL} / \mathrm{g}$ e o tipo $\mathrm{C}$, índice de expansão ao forno inferior a 12,2mL/g. O Estado de Minas Gerais (Figura 1 (c)) apresentou amostras classificadas como tipo B, com as menores expansões, entre 10,5 e $14,9 \mathrm{~mL} / \mathrm{g}$ e as amostras do Paraná apresentaram as maiores expansões, entre 12,2 e 16,5mL/g (Figura 1 (d)). Por esta análise, as amostras dos Estados de Santa Catarina e Paraná mostram maior uniformidade, com a distribuição dos índices de expansão seguindo uma distribuição normal ((b) e (d)), enquanto que as amostras do Estado de Minas Gerais foram visualmente mais desuniformes para este quesito de qualidade. A análise conjunta de todas as amostras (a) mostra um padrão de distribuição normal.

Como a expansão ao forno do polvilho azedo é a principal característica exigida por produtores e consu- 
midores, foi proposta uma classificação em nível nacional a partir da análise de classificação geral, onde o tipo A abrange os polvilhos com expansões superiores a $16,00 \mathrm{~mL} / \mathrm{g}$, tipo $B$ entre $12,00 \mathrm{~mL} / \mathrm{g}$ e $16,00 \mathrm{~mL} / \mathrm{g}$ e tipo $\mathrm{C}$ os polvilhos com expansões inferiores a $12,00 \mathrm{~mL} / \mathrm{g}$.

\section{4 - CONCLUSÕES}

A expansão ao forno do polvilho azedo pela metodologia prática, apresentou menor valor de variância em comparação àquela que usa Farinógrafo Brabender. Sendo simples e de baixo custo, é uma metodologia que se presta à implantação junto a empresas. Através dos resultados obtidos por esta metodologia é possível propor uma classificação em nível nacional dos índices de expansão ao forno das amostras de polvilho azedo. A proposta classifica como tipo A, o polvilho com qualidade extra e que apresente um índice de expansão ao forno superior a $16,0 \mathrm{~mL} / \mathrm{g}$, como tipo $B$ os polvilhos de média qualidade e que apresentem índices de expansão ao forno entre $12,0 \mathrm{~mL} / \mathrm{g}$ e $16,0 \mathrm{~mL} / \mathrm{g}$ e como tipo C os polvilhos de qualidade inferior que apresentem índices de expansão ao forno inferiores a $12,0 \mathrm{~mL} / \mathrm{g}$.

\section{5 - REFERÊNCIAS BIBLIOGRÁFICAS}

[1] AMERICAN ASSOCIATION OF CEREAL CHEMISTS INC. Approved methods of the American Association of Cereal Chemists. St. Paul, 1975.

[2] BENNION, E. B. Fabricación de pan. 4 ed: Ed. Acribia, p. 387-391, 1970.

[3] BRABET, C. et al Study of natural fermentation of cassava starch in Colombia. I: Characterization of the microflora and fermentation parameters. In: International Meeting on Cassava Flour \& Starch, Cali. Proceedings Cali: CIAT, p.120, 1994.

[4] CEREDA, M.P. Padronização para ensaio de qualidade da fécula de mandioca fermentada (polvilho azedo). I - Formulação e preparo de biscoitos. Boletim Sociedade Brasileira Ciência Tecnologia de Alimentos, v.17, n.3 p.287296, 1983a.

[5] CEREDA, M. P. Padronização para ensaios de qualidade da fécula de mandioca fermentada (polvilho azedo). II - En- saios de absorção de água. Boletim Sociedade Brasileira Ciência Tecnologia de Alimentos, v.17, n.3, p. 297 304, 1983b.

[6] CEREDA, M.P. Avaliação da qualidade de duas amostras de fécula fermentada de mandioca (polvilho azedo). Boletim Sociedade Brasileira de Ciência e Tecnologia de Alimentos, v.17 n.3, p.305-20, 1983c.

[7] CEREDA, M.P. Tecnologia e qualidade do polvilho azedo. Informe Agropecuário, v. 13, n. 145, p.63-68, 1987.

[8] CEREDA, M. P., NUNES, O. L. G. S. Definição de parâmetros de fermentação e secagem para industrialização do polviIho azedo. Botucatu: Departamento de Tecnologia dos Produtos Agropecuários, UNESP, 1989. 80 p. (Relatório Técnico, 1).

[9] DEMIATE, I. M.; SENGER, S. A.; VOGLER, Z.; CEREDA, M. P.; WOSIACKI, G. Característica de qualidade de amostra de polvilho azedo produzido ou comercializado no Estado do Paraná. Arquivos de Biologia e Tecnologia. Curitiba, v. 40, n. 2, p. 321-330, 1997.

[10] MESTRES, C.; ZAKHIA, N.; DUFOUR, D. Functional and physico - chemical properties of sour cassava starch. Starch Structure and Functionality/ Cambridge, UK: Royal Society of Chemistry, p. 42-50, 1997.

[11] NUNES, O.L.G.S.; CEREDA M. P. Metodologia para avaliação da qualidade de fécula fermentada de mandioca (polvilho azedo). In: Congresso Brasileiro de Mandioca, Salvador, 1994.

[12] NUNES, O. L. G. S. Efeito da radiação ultravioleta sobre as propriedades funcionais da fécula de mandioca tratada com ácido lático. Botucatu, 1994. 88p. Tese (Mestrado)Faculdade de Ciências Agronômicas, Universidade Estadual Paulista "Júlio de Mesquita Filho" (UNESP).

[13] PLATA-OVIEDO, M.; CAMARGO, C. R. O. Determinação de propriedades físico- químicas e funcionais de duas féculas fermentadas de mandioca (polvilho azedo). Ciência e Tecnologia de Alimentos, v. 4, n.1, p.59-65, Jan/Jun, 1995.

[14] TERNES, M.; MONDARDO, E.; VIZZOTTO, V. J. Variação do teor de amido na cultura da mandioca em Santa Catarina. Florianópolis, Secretaria da Agricultura e Abastecimento EMPASC, 1978. 22p. (Indicação de Pesquisa, 26).

[15] VILPOUX, O. Produção brasileira de polvilho azedo. Fax Jornal. CERAT, Botucatu: n. 47, p. 1-2, fev. 1997. 